in the internal environment that become more extreme with increasing age owing to decreased homoeostasis.

Of the 10 patients with post-hemiplegic epilepsy whose cases are described above six are still definitely living, one surviving for ten years. Of the four remaining cases Case 2 died two years after the onset of symptoms, Case 4 died six months after the onset of symptoms, and Case 6 died four years after the onset of symptoms. We have been unable to trace the fate of one of the remaining cases.

It is important to recognize that this condition responds extremely well to anticonvulsants.

Since becoming interested in this subject. I have found increasing numbers of epileptic syndromes occurring in this group of patients, and in view of the sequelae of intermittent confusion and paralysis the question of prophylactic anticonvulsants must be seriously considered in cases of hemiplegia secondary to cerebrovascular disease.

It will in due course be necessary to have extensive trials in order to discover whether such an approach could prevent unnecessary invalidism and hospitalization.

Finally a word about treatment. In the cases which I have so far treated phenytoin has been the drug of choice. This drug, even in small doses, has in most cases brought dramatic improvement. In one case of sensory epilepsy larger doses were needed. This patient had sensory signs, and conformed rather more closely with the concept of thalamic pain.

Since adopting this approach of anticonvulsant therapy to patients with post-hemiplegic pain I have so far not found anyone with this condition who did not respond to anticonvulsants.

Post-hemiplegic epilepsy occurs sometimes at night only. In this group phenobarbitone given at night is the drug of choice.

The use of phenobarbitone as a routine anticonvulsant in old people is likely to be fraught with the dangers of intoxication due to cumulation if there is impaired renal function.

\section{Conclusions}

Epilepsy in the elderly is a relatively common condition and and is usually caused by cerebrovascular disease.
Epilepsy occurring after hemiplegia may present with motor manifestations, sensory manifestations, or confusion, and may be misdiagnosed as a fresh cerebrovascular accident, thalamic pain, or senile dementia.

Post-hemiplegic pain, which has hitherto been regarded as intractable in many cases, may respond to treatment with anticonvulsant drugs.

It is suggested that the routine use of anticonvulsants in patients who have had cerebral thrombosis may in the future be regarded as justifiable.

\section{Summary}

Ten patients suffering from post-hemiplegic epilepsy are described. They fall into two clinical categories: (1) motor manifestations ; (2) sensorimotor manifestations.

Motor manifestations can be confused with fresh cerebrovascular accidents, and it is demonstrated that the sensory manifestations can be associated with pain severe enough to be confused with pain of thalamic origin.

The prognosis of post-hemiplegic epilepsy is discussed. It is emphasized that the occurrence of this condition, if recognized, is not necessarily of serious import provided the correct treatment is instituted. It is probable that post-hemiplegic epilepsy is relatively common.

Phenytoin is the drug of choice in treatment. Phenobarbitone as a routine anticonvulsant is contraindicated in old people. A routine course of anticonvulsants for patients who have had cerebral thromboses may in the future be regarded as justifiable.

\section{REFERENCES}

Bravais, L. F. (1827). Recherches sur les Symptomes et le Traitement de l'Epilepsie Hémiplégique. Paris.

Fine, W. (1966). Geront. Clin., 8, 121.

Gowers, W. R (1880). Lancet, 1, 315.

Penfield, W. (1933). Ann. intern. Med., 7, 303.

Russell, W. R. (1942). Brit. med. F., 2, 521.

Schwade E D. (1960). Geriatrics, 15, 11.

Tectures on Paralysis . . and Other Affections of the Nervous System, 2nd ed., p. 284 . London.

White, P. T., Bailey, A. A., and Bickford, R. G. (1953). Neurology (Minneap.), 3, 674 .

\title{
Outcome of Medically Treated Stroke Associated with Stenosis or Occlusion of the Internal Carotid Artery
}

\author{
PETER BRADSHAW,* M.D., M.R.C.P. ; EOIN CASEY,* M.B., B.SC.
}

\author{
Brit. med. 7., 1967, 1, 201-205
}

The recognition by angiography of stenosis or occlusion of the relevant internal carotid artery in patients with stroke has been followed by the introduction of various disobliterative procedures designed to prevent stenosis progressing to occlusion, to remove a potential site of emboli, and to restore normal carotid blood flow. Before it is possible to know the indications for or to evaluate the effects of such treatment, it is necessary to determine the usual outcome of stroke in patients treated medically or receiving no treatment. The purpose of this paper is to provide this information based on a study of 47 patients with stroke, of whom 24 had stenosis and 23 had occlusion of the relevant internal carotid artery.

Hunt (1914) first drew attention to the carotid vessels in the neck in connexion with hemiparesis, and Moniz, Lima, and
L'Acerda (1937) showed stenosis and occlusion of the relevant internal carotid artery by angiography in patients who had sustained stroke. Since then the clinical syndromes due to carotid insufficiency have been described by Johnson and Walker (1951), Fisher (1954), Smyth (1954), Symonds (1957), and others. Four modes of clinical presentation have been recognized. The first, transient cerebral ischaemic attacks, is characterized by attacks of recurrent unilateral weakness or paraesthesiae, which usually first affect the hand, later the upper and lower limbs, and occasionally the face. These episodes tend to become more profound, more extensive, and longer-lasting, and either abate spontaneously or terminate in lasting hemiplegia.

* Neurological Department of the United Sheffield Hospitals. 
Transient ipsilateral blurring or loss of vision with or without limb symptoms, ingravescent hemiparesis, or sudden stroke may occur less often. The condition may be suspected when a bruit is heard over the appropriate side of the neck or when pressure in the ipsilateral retinal artery, measured by ophthalmodynamometry, is reduced (Marshall, 1960). Elkington (1958) noted that the ratio of men to women was $3: 1$, that symptoms usually began during or after the fifth decade, and that many of these subjects had hypertension or peripheral vascular disease.

Little has been written about the prognosis for stroke associated with occlusive carotid lesions. Hurwitz, Groch, Wright, and McDowell (1959) followed up 57 patients who had experienced transient cerebral ischaemic attack, and after one year all were neurologically normal. However, the presence of carotid lesions in many had not been verified by angiography. Hardy, Lindner, Thomas, and Gurdjian (1962) followed up 55 patients who had stroke and radiographically proved occlusive carotid disease. After an average period of 3.7 years they found that 33 had died, and of the survivors $11 \%$ were normal, $11 \%$ improved, $27 \%$ unchanged, and $51 \%$ had deteriorated. Marshall (1964) discussed the prognosis for transient cerebral ischaemic attacks and observed that $60 \%$ of patients so afflicted became seriously disabled during their first or second attack, and in 16 of the 34 who were followed up this ensued within one month of onset.

\section{Selection of Patients and Clinical Methods}

Between January 1956 and September 1965, 47 patients with stroke were admitted to the department of neurology of the United Sheffield Hospitals and shown by arteriography to have occlusive lesions of the appropriate internal carotid artery. These included 24 with stenosis and 23 with occlusion, of whom two also had stenosis of the opposite internal carotid artery. During the earlier years covered by this study a number of similar patients were admitted to the department but were rejected because either the radiographs failed to show the needle within the lumen of the common carotid artery or provided inadequate pictures of the carotid lesion. Also, we were reluctant to submit the elderly to angiography, and only two patients over the age of 65 have been included, while others who had serious cardiac disease or gross peripheral atheroma were thought to be unsuitable for arteriography and were excluded.

Initial assessment was made personally, and particular attention paid to the state of the peripheral arteries and retinal arterioles. Hypertension was accepted as a diastolic blood pressure persistently above $90 \mathrm{~mm}$. in men and $100 \mathrm{~mm}$. in women. The designation of neurological disability was made as follows:

Normal.-Asymptomatic and without neurological signs.

Minimal Residua.-Asymptomatic with no more than slight reflex asymmetry and/or feeble extensor plantar response.

Slight Disability.-Normal speech associated with clumsiness, slight spasticity, and relative weakness, with appropriate reflex change compatible with full-time light employment.

Moderate Disablement.-Occasional nominal dysphasia, and/or moderate spastic weakness of the limbs incompatible with full-time light employment but ability to walk 500 yards ( 460 metres) with a toe-raising spring and/or a stick and to lift a cup to the lips with the affected hand.

Severe Disablement.-Any disability greater than the above.

The following investigations were carried out on all patients: full blood count, erythrocyte sedimentation rate (E.S.R.), urinalysis, plain radiography of chest and skull, and a blood Wassermann reaction (W.R.). The cerebrospinal fluid (C.S.F.) was examined in 32 patients. Percutaneous carotid arteriography was carried out bilaterally in eight patients and on the appropriate side only in 37 . Two others had an arch aortogram - one by a catheter inserted into the left femoral artery, the other via the right brachial artery. In both of these the relevant vessels in the thorax and neck were visualized and afterwards appropriate percutaneous arteriography was done to obtain clear pictures of the intracranial circulation. Carotid stenosis was assessed from the radiographs and was termed severe when the arterial lumen was narrowed by two-thirds or more, moderate when it was one-third to two-thirds, and slight when less than one-third.

Five patients died under our care-two during their first and three during their second admission to hospital. The causes of death were verified at necropsy. The remainder were followed in the first instance for four to eight months. Between January and March 1966 an attempt was made to trace the survivors. It was found that four patients had died; the causes of death in two had been verified elsewhere and details of the terminal illness were supplied in the other two by the family doctor. Of the 37 survivors 35 were examined personally, and in two postal information was supplied both by the patient and by the family doctor. The final state was regarded as satisfactory when the patient was normal or had minor residua or slight disability.

\section{Findings}

Table I shows the age at which neurological symptoms began and the sex distribution. It will be seen that the average age at onset was 55 and the ratio of men to women was about $3: 1$ in both carotid stenosis and occlusion. All but six patients were examined within three months of the start of their illness. Thirty-four patients had arterial hypertension, and in seven the diastolic pressure was persistently over $115 \mathrm{~mm}$. One normotensive and 18 hypertensive patients showed evidence of peripheral atheroma consisting of tortuosity and thickening of limb arteries ; 14 also had impairment of peripheral pulses, five cold blue feet, and one gangrene of one toe. In addition, 11 of these patients displayed marked irregularity in the calibre of the retinal arterioles, and this was also detected in three others who had no other evidence of peripheral arterial disease. Angina pectoris was a symptom in four patients, three of whom had peripheral atheroma. Overall 35 patients had either hypertension or peripheral atheroma or both.

\begin{tabular}{|c|c|c|c|c|c|}
\hline & & Males & Females & Age Span & Average Age \\
\hline \multirow[t]{2}{*}{$\begin{array}{l}\text { Carotid stenosis } \\
, \quad \text { occlusion }\end{array}$} & $\begin{array}{l}\cdots \\
\cdots\end{array}$ & $\begin{array}{l}19 \\
17\end{array}$ & $\begin{array}{l}5 \\
6\end{array}$ & $\begin{array}{l}35-69 \text { years } \\
44-66 \text { " }\end{array}$ & $\begin{array}{l}55 \text { years } \\
55 \%\end{array}$ \\
\hline & & 36 & 11 & $35-69$, & 55 \\
\hline
\end{tabular}

\section{Symptoms and Signs}

Thirty-three patients presented with transient cerebral ischaemic attacks which lasted for between 30 minutes and 24 hours. These consisted of transient unilateral weakness or paraesthesiae affecting one or both limbs in 31, and eight of these also experienced monocular visual disturbance. Two others, who made no complaint of limb symptoms, had episodic confusion and dysphasia. Sudden stroke occurred in 11 patients and in three there was ingravescent hemiparesis which had increased during a period of 24 hours or more.

Table II shows the extent of initial disability; it will be seen that 16 of the 24 patients with carotid stenosis were either normal or had only slight disability, whereas 18 of the 23 patients

TABLE II-Grade of Disability at Initial Assessment

\begin{tabular}{|c|c|c|c|}
\hline & Carotid Stenosis & Carotid Occlusion & Total \\
\hline $\begin{array}{l}\text { Normal . } \\
\text { Slight disability } \\
\text { Moderate disablement } \\
\text { Severe }\end{array}$ & $\begin{array}{l}8 \\
8 \\
3 \\
5\end{array}$ & $\begin{array}{r}0 \\
5 \\
12 \\
6\end{array}$ & $\begin{array}{r}8 \\
13 \\
15 \\
11\end{array}$ \\
\hline
\end{tabular}


with internal carotid occlusion had moderate or severe disablement.

A neck bruit, detected in 12 patients, was audible over the appropriate side of the neck in nine and was heard on both sides in three. Dysphasia was present in 17 patients (severe in three, moderate and chiefly expressive in eight, and in six consisting of an occasional nominal defect); in all there was other cause to incriminate the dominant hemisphere. Thirty-four patients displayed unilateral pyramidal-tract signs, consisting in spasticity, weakness, and appropriate reflex change. Involvement of the face was uncommon and seldom amounted to more than slight relative weakness; the upper limb was usually most severely involved, though in 28 patients the lower limb was also affected. Sensory impairment when present involved approximately the same territory as the weakness, and consisted in relative cutaneous sensory impairment in 22 and tactile inattention in three. The frequency of other abnormalities was as follows: mild intellectual impairment (5), ipsilateral optic atrophy and blindness (1), contralateral homonymous hemianopia (1), and wasting of the small muscles of the hand associated with parietal lobe sensory disturbance (1).

\section{Investigations}

Plain radiographs of chest were normal in 32 patients and showed moderate cardiac enlargement in 11 , mitral stenosis in one, arrested apical pulmonary tuberculosis in one, and mild pneumoconiosis in two.

Plain radiographs of skull were normal in 44 patients, and in three showed faint calcification in the vicinity of the carotid siphon.

A full blood count, E.S.R., and urinalysis were normal and the blood W.R. was negative in all 47 patients.

The lumbar C.S.F. pressure, manometrics, and constituents were normal and the W.R. negative in 32 patients.

An electrocardiogram was taken in 31 patients and was normal in 23 ; left ventricular hypertrophy was revealed in seven, chronic ischaemic change in two, and a recent posterior infarction in one.

The plasma urea and electrolytes were estimated in 18 patients, including all seven with moderate to severe hypertension, and were normal.

Cerebral angiography.-The carotid circulation was examined in 10 patients on both sides, and in three bilateral abnormalities were detected. Two patients had carotid occlusion of the appropriate side and stenosis of the opposite side respectively in the siphon and adjacent to the carotid canal ; one other had stenosis of the appropriate side and an intracavernous carotid aneurysm of the other. The occlusive lesions were located on the right in 17 , on the left in 28 , and bilaterally in two. The site of the lesion was within the proximal $2 \mathrm{~cm}$. of the internal carotid artery in 44 , adjacent to the carotid canal in two, and in the siphon in three. The extent of the stenosis detected in 24 patients was severe in five, moderate in eight, and slight in 11. In four patients with carotid stenosis abnormalities of the ipsilateral intracranial circulation were detected as follows: anterior cerebral stenosis (1), middle cerebral stenosis (1), and middle cerebral occlusion (2).

Three patients, all of whom had severe carotid stenosis, slight disability, and peripheral atheroma, sustained serious complications within two hours of percutaneous carotid arteriography. One of these died five days later of cerebral oedema due to middle cerebral embolism, shown at necropsy to have arisen from fresh thrombus attached to an area of carotid stenosis, and two others became severely hemiplegic within one hour of the procedure. In one of these there was an abnormally low carotid bifurcation into which the needle had been inserted; in the other a severe stenosis became occluded between the first and the second injection.

\section{Treatment}

Thirty-three patients received only supportive treatment. Three patients with carotid stenosis and four with carotid occlusion had blood pressures of between 250/130 and 200/115 $\mathrm{mm}$. ; these were reduced over the course of one to three weeks with methyldopa to levels of $180 / 100$ to $170 / 90$, which were maintained throughout the period of observation. No complications of this therapy were encountered. Five other patients received anticoagulants, including heparin for 48 hours and phenindione (Dindevan) for three to eight weeks. This number included all who sustained complications of angiography, one with progressive stroke, and one who had multiple transient cerebral ischaemic attacks. Stabilization was effected in hospital, and prothrombin times of about double that of fresh controls were maintained and checked after discharge from hospital by weekly tests. Care was taken to taper off treatment over a period of weeks.

\section{Progress}

Carotid Stenosis.-Two patients died from related diseaseone from middle cerebral embolism after angiography and the other from verified cerebral haemorrhage 15 months after the onset of stroke. One patient survived stroke for five years and nine months, during which time he was normal, but then succumbed to renal tumour. The 21 survivors were assessed after intervals of six months to eight years ; 11 were normal, one had minor residua, two slight disability, and one moderate and six severe disablement. Fig. 1 shows the duration of the follow-up and

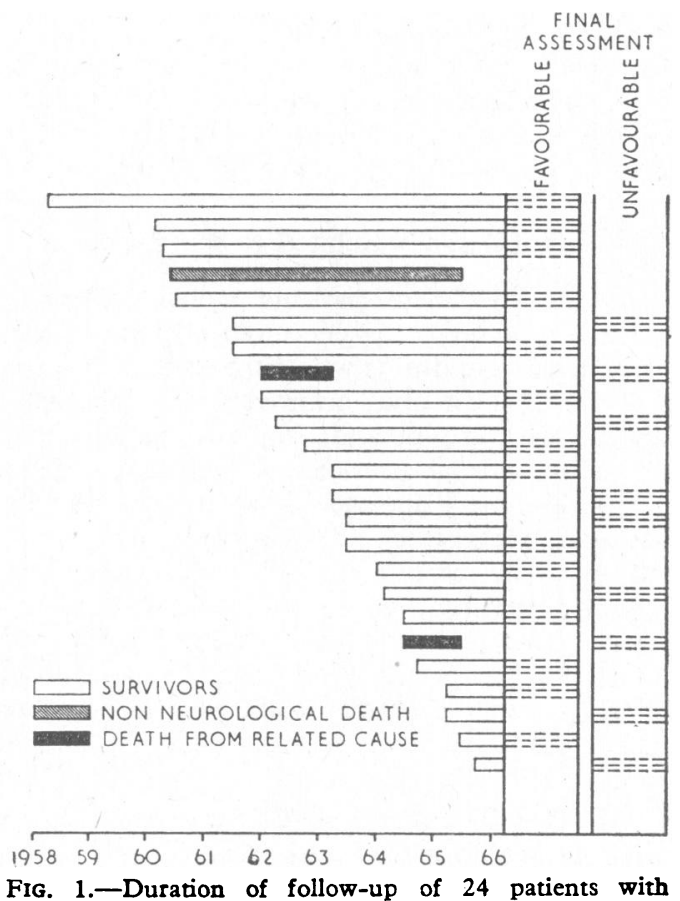

Fig. 1.-Duration of follow-up of 24 patients with
internal carotid stenosis and results of final assessment.

whether final assessment was satisfactory-that is, normal, minor residua, or slight disability-or was unfavourable.

Carotid Occlusion.-Five patients died of related disease: one from pulmonary embolism, one from thrombosis of the opposite internal carotid artery, and one from verified and two from unverified cerebral haemorrhage. Though the four lastmentioned failed to survive to the end of the period of observation, two lived to the ages of 69 and 73 and two survived stroke by three and four years respectively with only slight disability. Two patients died from unrelated conditions-one recovered from his stroke and was normal until shortly before his death from bronchial carcinoma five years later, the other survived 
five years with slight disability and died from rectal carcinoma. The 16 survivors were followed for one to ten years, when two were normal, two had minor residua, five had slight disability, and three moderate and four severe disablement. Fig. 2 shows the duration of the follow-up and whether the outcome of stroke was satisfactory or unfavourable.



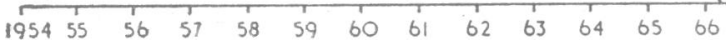

FIG. 2.-Duration of follow-up of 23 patients with internal carotid occlusion and results of final assessment.

All 14 patients, seven with stenosis and seven with occlusion of the internal carotid artery, who survived to the end of the follow-up but who failed to make substantial recovery improved.

\section{Clinical Data Related to Prognosis}

Carotid Stenosis.-These patients were divided into two groups: the 15 who from the neurological point of view made satisfactory recovery, and nine who either died of related disease or failed to make substantial improvement. The average age was 54 in the first group, and only two patients had either irregularity of the retinai arterioles or peripheral atheroma ; in the second group the average age was 64 and seven had evidence of peripheral arterial disease. The prognosis was not related to the number of transient cerebral ischaemic attacks before first assessment, hypertension, or cardiac enlargement or angina pectoris. Only one patient who presented with more than slight disability and one with more than slight stenosis made a good recovery, while no patient with stenosis or occlusion of intracranial arteries ipsilateral to carotid stenosis made substantial improvement.

Carotid Occlusion.-Patients were divided in a similar fashion into groups of 11 who made satisfactory neurological progress and 12 who either died from related disease or failed to make a good recovery. The average age of the first was 51 , and only two had either irregularity of the retinal arterioles or peripheral atheroma. In the second group the average age was 55, and 11 had peripheral arterial disease. There was no relation between prognosis and hypertension, cardiac enlargement, angina pectoris, or the number of transient cerebral ischaemic attacks before the first examination.

\section{Discussion and Conclusion}

The clinical and radiological findings of 47 patients with stroke are presented, 24 of whom had stenosis and 23 occlusion of the appropriate internal carotid artery. These differ in no important way from those reported in other medical and surgical series. The present study has largely excluded the elderly and many with gross peripheral circulatory disturbance or serious cardiac disease and makes no claim to have provided a comprehensive survey of the condition. Nevertheless, it is likely that surgeons concerned with the treatment of the malady have exercised a similar selection.

The outcome of stroke in 24 patients with internal carotid stenosis was that two died of related and one from unrelated disease, though the last-mentioned was normal for nearly five years after his stroke. The 21 survivors were followed for between six months and eight years, when 11 were normal, one had minor residua, two slight disability, and one moderate and six severe disablement. Thus from the neurological point of view the outcome was satisfactory in $65 \%$ and unfavourable in $35 \%$. These results are marginally better than was reported to follow disobliterative surgery by Edwards, Gordon, and Rob (1960) but are inferior to those of Kenyon and Thompson (1965), who stated that 30 out of 55 patients followed for 1 to 11 years were normal. The initial disability of our patients was greater overall than in Kenyon and Thompson's patients. It should be noted that the series of De Bakey, Crawford, Cooley, Morris, Garrett, and Fields (1965) included some patients who had neither neurological symptoms nor signs prior to disobliterative carotid surgery, and therefore cannot be contrasted with the present study.

The outcome of stroke in 23 patients with internal carotid occlusion was that five died of related and two from unrelated disease, though one of the latter was neurologically normal for four years and the other survived with slight disability for five years. The 16 survivors were followed for between one and ten years, when two were normal, two had minor residua, five slight disability, and three moderate and four severe disablement. Thus from the neurological point of view the outcome was satisfactory in $45 \%$ and unfavourable in $55 \%$. These results are better than have been reported to follow disobliterative surgical treatment by Lemmen, Davis, and Radnor (1958), Edwards et al. (1960), and Hardy et al. (1962). Few patients who develop severe disablement may make any substantial recovery, and the problem posed is to determine the likely prognosis before this event.

The prognosis is unrelated to the number of preceding transient cerebral ischaemic attacks, hypertension, cardiac enlargement, or angina pectoris. There is, however, an association between the presence of either marked irregularity in the calibre of the retinal arterioles or peripheral atheroma and a poor prognosis. Such abnormalities were found in eight out of nine patients with carotid stenosis and in 11 out of 12 with occlusion who either died from related disease or failed to make satisfactory progress, and in only 2 out of 15 with stenosis and in 1 out of 11 with occlusion who did well. It would seem that patients with little or no disability who have transient cerebral ischaemic attacks and who have healthy peripheral arteries are in no great peril, and it is this same group who are reported to show the best response to surgical treatment.

The dangers of percutaneous carotid arteriography are evident, especially when there exists a severe degree of stenosis and there is peripheral atheroma. One such patient died and two others became severely hemiplegic within two hours of the procedure and have remained disabled. Since this study was closed a further two similar patients have suffered severe disablement after angiography. Though we have limited experience of cerebral angiography carried out by means of a catheter inserted remote from the carotid circulation, described by Burrows and Marshall (1965), this method would seem to avoid many of the hazards of direct needle puncture. When by this means a moderate or severe degree of carotid stenosis is revealed, especially in a man with a poor peripheral circulation, then medical treatment is unlikely to prevent severe disablement and surgery may have more to offer. However, if the intracranial arteries ipsilateral to carotid stenosis are stenosed 
or occluded, then the damage has already been done and restoration of normal carotid blood flow is unlikely to achieve clinical improvement.

\section{Summary}

The clinical and radiological features of 47 patients with stroke are presented, of whom 24 had stenosis and 23 occlusion of the relevant internal carotid artery.

One patient died and two became hemiplegic after percutaneous carotid arteriography.

Thirty-three patients had only supportive treatment, five received anticoagulants for three to eight weeks, and hypertension was reduced in seven with methyldopa.

Two patients with carotid stenosis died of related and one of unrelated disease. The survivors were followed for between six months and eight years. Overall, neurological progress was highly satisfactory in $65 \%$ and unfavourable in $35 \%$.

Five patients with carotid occlusion died of related and two of unrelated disease. The survivors were followed for between one and ten years. Overall, neurological progress was highly satisfactory in $45 \%$ and unfavourable in $55 \%$.

The presence of marked irregularity in the calibre of the retinal arterioles and/or atheroma of limb vessels was usually associated with a poor prognosis.
ADDENDUM.-The progress of all survivors in this series has been followed up to 1 November 1966. One patient with carotid stenosis previously classified as having moderate disablement has improved and now has only slight disability, the remainder are unchanged.

\section{REFERENCES}

Burrows, E. H., and Marshall, J. (1965). F. Neurol. Neurosurg. Psychiat. 28, 533 .

DeBakey, M. E., Crawford, E. S., Cooley, D. A., Morris, G. C., Garrett, H. E., and Fields, W. S. (1965). Ann. Surg., 161, 921

Edwards, C. H., Gordon, N. S., and Rob, C. (1960). Quart. F. Med., 29, 67.

Elkington J St C. (1958) Lancet, 2, 275, 327

Fisher, M. (1954). Arch. Neurol. Psychiat., 72, 187

Hardy, W G Lindner, $D$. W. Thomas, L. M., and Gurdiian, E. S. (1962). Arch. Neurol., 6, i38.

Hunt, J. R. (1914). Amer. F.' med. Sci., 147, 704

Hurwitz, L. J., Groch, S. N., Wright, I. S., and McDowell, F. H. (1959) Arch. Neurol., 1, 491

Johnson, H. C., and Walker, A. E. (1951). 7. Neurosurg., 8, 631

Kenyon, J. R., and Thompson, A. E. (1965). Brit. med. 尹., $1,1460$.

Lemmen, L. J., Davis, J. S., and Radnor, L. L. (1958). F. Neurosurg. $15,438$.

Marshall, J (1960). Trans, ophthal Soc (U.K.) 80, 263.

Marshal1, 1964). Quart. F. Med., 33, 309.

Moniz E4) Quéd. 45, 977.

Moniz, E., Lima, A., and L'Acerda, R. de (1937). Pres.

Symonds, C. P. (1957). In Modern Trends in Neurology, 2nd series, edited by $\mathrm{D}$. Williams. London.

\title{
Glycosuria of Pregnancy
}

\author{
J. FINE,* M.D., D.P.H., D.T.M.
}

Brit. med. 3., 1967, 1, 205-210

The present communication is an inquiry into the incidence and significance of the glycosuria of pregnancy.

Though there have been many contributions to the literature of pregnancy glycosuria since the beginning of the century, a re-examination of the subject is desirable for a number of rcasons. First, only recently have specific methods become available for the detection and estimation of glucose in urine, so that much of the older work has become invalidated. Secondly, a full appreciation of the incidence of pregnancy glycosuria is possible only by comparison with incidence studies carried out in the general population: no such comparative investigations have yet been attempted. Thirdly, though the consensus of opinion is at present against any relationship of the glycosuria to diabetes, formal proof of this is lacking, and there are still many clinicians who believe that it may have at least prediabetic significance. In this area hundreds of glucose-tolerance tests have been performed in the past few years on cases presenting glycosuria at the antenatal clinic to ensure that no diabetic is being overlooked, and longterm follow-ups of such cases are being planned. Josling et al. (1952) treated the finding of glycosuria with the utmost seriousness.

It might be asked-and it should be asked-how justified is this energetic approach to the problem in the present state of knowledge of the subject. It was in the hope of answering this and other questions in the field of pregnancy glycosuria that the investigations described below were undertaken.

\section{Reports of Glycosuria Incidence}

There is a considerable divergence of opinion about the extent of glycosuria in pregnancy. Payer (1899) found an incidence of $50 \%$, but much lower' figures have been reported since. Brews (1963) found that $33 \%$ of urines of pregnant women gave reduction with Benedict's solution, while Peel (1963) states that approximately $30 \%$ show glycosuria on occasion: a figure of $20 \%$ is given by Johnstone and Kellar (1963), Cantarow and Trumper (1962) give a range of $15-25 \%$, Harrison ct al. (1962) 10-15\%, and Williams (1925) $13.6 \%$. A number of writers give much lower figures: $6.3 \%$ by Gross et al. (1956), 5.4\% by Williams and Wills (1929), and $5 \%$ by Bodansky and Bodansky (1952). The lowest value reported is by Adair (1940), who found only $3 \%$.

\section{Preliminary Investigation}

A series of 1,547 patients attending the antenatal clinic at the maternity department of the County Hospital, Griffithstown, Monmouthshire, were investigated by means of the conventional method of Clinistix combined with Benedict's qualitative test. On a first examination 145 cases $(9.3 \%)$ were positive for urine glucose. When further specimens from a number of patients were tested the total showing glycosuria on one or other occasion rose to $251(16.2 \%)$. It seemed reasonable to suppose that had all the cases been examined more than once a still higher percentage of those with glycosuria would have been obtained.

The above figures throw some light on the diversity of reports on glycosuria incidence: a combination of the intermittency of the glycosuria and a variation in the number of specimens per patient examined could partly explain it. Another factor is the nature of the method employed in urine-testing: thus

\footnotetext{
- Senior Consultant Pathologist, Royal Gwent Hospital, Newport, Mon. At present: Pathologist, Kitwe Central Hospital, Kitwe, Zambia. 